\title{
Digital Topology Java Applet
}

\author{
Carmen Escribano \\ Antonio Giraldo \\ María Asunción Sastre \\ Mario Trotta \\ 34913365474 \\ 34913365474 \\ 34913365474 \\ 34655411965 \\ cescribano@fi.upm.es \\ agiraldo@fi.upm.es \\ masastre@fi.upm.es mtrottam@hotmail.com
}

Computer Science School, Technical University of Madrid, Boadilla del Monte, 28660 Madrid, Spain

\begin{abstract}
We present here a java applet, accessible through the World Wide Web, which allows to apply to a binary digital image a series of topological algorithms for image processing.
\end{abstract}

\section{Categories and Subject Descriptors}

I.4.6 [Image processing and computer vision]: Segmentation I.4.8 [Image processing and computer vision]: Scene analy sis

\section{General Terms}

Algorithms.

\section{Keywords}

Digital Topology, Image Processing, Thinning.

\section{DESCRIPTION OF THE WORK}

A binary digital image $P$ can be represented as a set of pixels: a set of black pixels representing the image, and a set of white pixels for the background. Moreover, in order to connect the pixels of the image, two different adjacency relations can be consider: 8-adjacency and 4-adjacency, where two pixels are 4adjacent if they share a common side, while they are 8-adjacent if they share a common side or a common vertex.

These adjacency relations provided the fundamental structure to define several important image processing operations which we have implemented in a java applet. All algorithms included in it have two different versions according to the adjacency relation considered.

These algorithms are the following:

1. Basic operations on digital images.

1.1. Counting and labeling components.

1.2. Finding boundaries.

1.3. Finding boundary curves: Starting from a pixel in the boundary, the algorithm goes round the boundary generating a chain code.

1.4. MAT (Medial Axis Transform): This is the set of pixels which are center of maximal circles contained in the set of black pixels. To define these circles, we can consider two different distances: distance D4 is the sum of the horizontal and vertical distances, while distance D8 is the biggest of the horizontal and vertical distances.

\section{Thinning algorithms}

Sequential thinning: The image is scanned downwards and from left to right. A pixel is deleted if it is in the boundary, it is not an end pixel of a thin line and its deletions does not alter the connectivity of the image.

2.1. Parallel thinning of north boundary pixels: An adaptation of the previous algorithm suitable for a parallel processing.

2.2. Parallel algorithm ROS: Similar to the previous one alternating between north, west, south and east boundary pixels.

2.3. Parallel thinning using subcycles: Each iteration of the algorithm has two subcycles in which different kind of pixels are deleted.

2.4. Algorithm GH89-A2: It alternates between two subfields of the image which would correspond, respectively, to the black and white squares of a chess board.

2.5. Medial axis: It computes a skeleton of the image, which consists on the set of pixels whose distance to the boundary are local maxima.

2.6. Iterative thinning via boundary generation.

This applet is used as an interactive learning tool in the course "Digital Topology" taught at the Computer Science School of the Technical University of Madrid. The course and the applet can be accessed at the following internet addresses:

www.dma.fi.upm.es/docencia/segundociclo/topologiadigital www.dma.fi.upm.es/java/topologiadigital/applet

Most of the algorithms included in the applet can be found in [5] We recommend $[1,4,6]$ for information on Digital Topology, and $[2,3]$ for the broader field of Digital Geometry.

\section{REFERENCES}

[1] Escribano C., Giraldo A. and Sastre M.A., Topología Digital, FGUPM, 2007.

[2] Klette R. and Rosenfeld A., Digital Geometry, Elsevier, 2004.

[3] Herman G.T., Geometry of digital spaces, Birkhauser, 1998.

[4] Kong T.Y. and Rosenfeld A., Digital Topology: Introduction and survey, Computer Vision, Graphics and Image Processing 48 (1989) 357--393.

[5] Kong T.Y. and Rosenfeld A. (eds.), Topological algorithms for digital image processing, Elsevier, 1996.

[6] Rosenfeld A., Digital Topology, American Mathematical s 